\title{
Microwave Biological Effects and Research Progress
}

\author{
Yiou Cheng', Kai Kang,a \\ ${ }^{1}$ School of Electronic Engineering, University of Electronic Science and Technology of China, Chengdu \\ 611731, China \\ akangkai@uestc.edu.cn
}

Keywords: microwave; biological effects; functional mechanism; research progress

\begin{abstract}
The study on the biological effects of electromagnetic field has received extensive attention since the 18th century. As a kind of electromagnetic wave, microwave has the frequency of $300 \mathrm{MHz} 300 \mathrm{GHz}$. Among the studies concerning microwave biological effects, the study about the mutual relationship between electromagnetic field and life has an important status. Together with the constant deepening of relevant studies, people have more in-depth understanding about the properties of microwave and the electromagnetic properties of organism. Meanwhile, great progress has been made in the study about microwave biological effects. This thesis starts from the perspective of microwave biological effects to analyze its functional mechanism and present research progress.
\end{abstract}

\section{Introduction}

The biological effects of microwave could be reflected from multiple aspects. The most direct reflection is the damage brought about by microwave radiation to organism. Accordingly, a series of resulting epidemic diseases have obtained the wide concentration from all walks of life. The study on microwave biological effects and corresponding functional mechanism contributes to the prevention of possible harms caused by microwave to human body. In recent years, with the deepening of relevant studies, people have new insights about the functional mechanism of microwave biological effects. Therefore, it is necessary to make summary about present research progress and therefore promote the further deepening of relevant studies.

\section{Biological Effects of Microwave}

\section{Epidemic Diseases Triggered by Microwave Radiation}

As proved by relevant studies, workers who have close contact with microwave radiation have higher occurrence of neurosism, lens opacity, cardiovascular disease and leukemia than common people. For instance, the occurrence rate of neurosism among pulse microwave contactees reach up to $40.3 \%$. In continuous microwave working environment, the occurrence rate of neurosism among the working staff also reaches up to $37.1 \%, 5.1 \%$ higher than that in non-microwave radiation environment[1]. Specific investigation statistics are shown in Table 1. Relevant researchers also conduct the survey on the harms of microwave on human crystalline lens, finding that its corresponding occurrence rate is far above than that in control group. In addition, people engaged in television maintenance and television broadcasting have higher occurrence rate of cardiovascular disease and leukemia than common people[2]. This proves that microwave is the principal cause of these diseases[3]. 
Table 1 Study on the Influences of Microwave on Neurosism Occurrence Rate

\begin{tabular}{|c|c|c|c|}
\hline experiment group & $\begin{array}{c}\text { microwave } \\
\text { frequency } \mathrm{MHz}\end{array}$ & $\begin{array}{l}\text { power } \\
\mathrm{mW} / \mathrm{cm} 2\end{array}$ & occurrence rate \\
\hline control group & 1 & 1 & $5.1 \%$ \\
\hline $\begin{array}{l}\text { pulse microwave } \\
\text { environment }\end{array}$ & $400 \sim 9400$ & $0.07 \sim 0.18$ & $40.3 \%$ \\
\hline $\begin{array}{l}\text { continuous } \\
\text { microwave } \\
\text { environment }\end{array}$ & 3400 8600 & $0.06 \sim 0.15$ & $37.1 \%$ \\
\hline
\end{tabular}

\section{Influences of Microwave on Biological Cells}

The Influences of microwave on biological cells are mainly presented in the following few aspects[4]. First of all, microwave could accelerate the cell apoptosis. Secondly, microwave radiation will change the cell membrane permeability of biological cells and film potential, trigger electroporation effects, and lead to cytoplasm injection and entrance of other matters. Thirdly, microwave has influences on cell ion channel, including sodium, potassium and calcium. Throughout the radiation experiment on lymphocyte with $2450 \mathrm{MHz}$ continuous microwave, the biphasic phenomena of calciuk ion concentration and the correlation between dose and time as well as time and effects could be observed. $72 \mathrm{MHz} 10 \mathrm{GHz}$ microwave will change the flow of sodium and potassium ions in the cell membrane. Fourthly, microwave will change the chromosome structure and functions of cells, including the damage repair ability and tolerance of cell mutation. Fifthly, microwave could change the signal transduction in the cells and influence trans-membrane information expression and cell gap communication ability. Finally, microwave could change cell enzymatic activity. Specific influences are as shown in Table 2.

Table 2 Influences of Microwave on Cell Enzymatic Activity

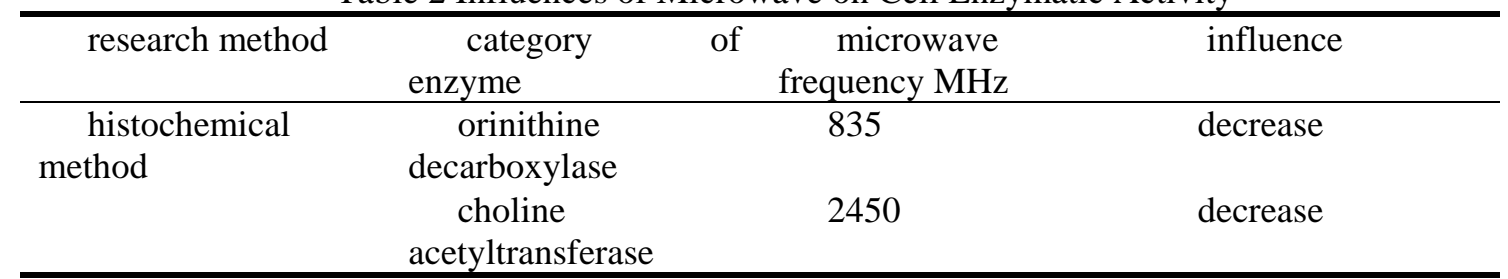

\section{Influences of Microwave on Organism Growth Rate}

The influence of microwave on organism growth speed is mainly reflected from growth rate resonance effects. Although this study has been testified by many previous studies, the growth speed of organism is also under the influence from other unknown elements in addition to microwave. According to present research progress, the influence of microwave is mainly expressed by the reliance of organism growth on microwave frequency modulation. In addition, microwave has different influences on different cells. At the same time, the synchronization effects of microwave on organism growth could be observed.

\section{Research Progress of Microwave Biological Effect Functional Mechanism}

\section{Microwave Properties}

The ionizing radiation of electromagnetic wave includes X-ray, Y-ray and light radiation. Other elements including microwave belong to non-ionizing radiation. The ionizing radiation will force the fracture of molecular chain. Instead, non-ionizing radiation will not generate influences on chemical bonds.

\section{Organism Electromagnetic Properties}

Organism is a diversified composition structure. As a composite medium, the electromagnetic properties of organism mainly contain electrical conduction property and dielectric property. Since organism is always in an unbalanced medium state, temperature, time and physical factors all generate influences on electromagnetic parameter changes. Moreover, the psychological activities of organism indeed originate from the electromagnetic properties. As a result, due to the great 
complexity of organism electromagnetic properties, existing study could only start from a macroscopic perspective to observe organism properties. According to the analytic results from this perspective, it could be founded out that organism dielectric property is the response of molecular bound charge in organism on external electric field. Dielectric coefficients are often influenced by molecular structure, external electric field time rate of change and temperature. If external electric field is the time-harmonic field, then dielectric coefficient is related to frequency. The electrical conductivity property of organism is the response of internal free charge on external electric field. There also exists non-linear electric phenomenon.

\section{Microwave Thermal Effects}

Microwave effects symbolize the influences of thermal energy transformed from electromagnetic energy on organism. Such effects are mainly reflected from molecular thermal movement effects. When the temperature of organism arises, thermal movement effects present linear response properties within $0 \sim 105 \mathrm{~V} / \mathrm{m}$ field range. If organism cells are compared as the point termination containers under the influences from electric field, then electric diphole in cellular tissues would produce displacement current and generate considerable heat and conductivity current out of the fraction and collision with surrounding particles. Accordingly, the electric medium heats up. Moreover, the body fluid within the organism will form closed loop. Organism will heat up together with the formation of partial response vortex.

\section{Microwave Non-Thermal Effects}

The non-thermal effects of microwave on organism refer to a series of physiological and pathological reactions generated in organisms under low-power microwave radiation, which will be carefully presented in the following four aspects. Firstly, non-thermal effects are always accompanied by thermal effects. Secondly, organism electromagnetic properties have uneven properties. In spite of the rise of temperature in partial parts of organism under microwave radiation, the overall changes of the organism are less prominent. Thirdly, due to the poor repetition, non-thermal effects could not be easily captured. Fourthly, non-thermal effects usually take place in balanced state. The comparison of microwave thermal effects and non-thermal effects is presented in Table 3.

Table 3 Comparison of Functional Mechanism of Microwave Thermal Effects and Non-thermal Effects

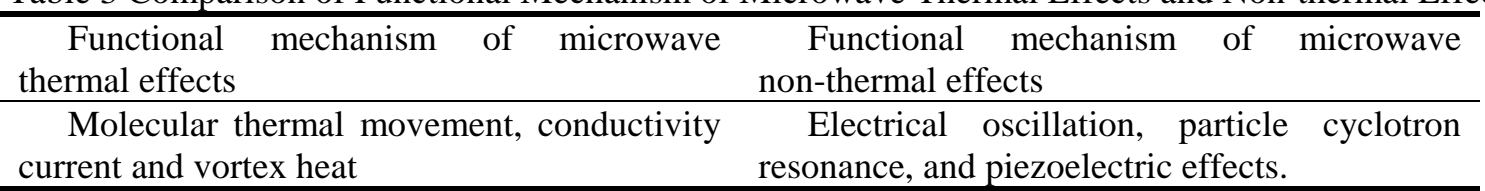

\section{Other Biological Effects of Microwave}

As a general rule, although relevant study concerning microwave biological effects has made great progress, there still lacks a uniform conclusion about functional mechanisms. Besides above-mentioned biological effects, there have cumulative effects, distant effects, delayed effects and amplification effects. Therefore, the study about microwave biological effects has greater development space. At present, microwave has been widely applied in the field of food industry and medicine industry, in particular microwave retrieval and sterilization. At the same time, this provides references for prevention and control of electromagnetic pollution.

\section{Conclusion}

To sum up, microwave biological effects possess diversified influences in multiple aspects, ranging from the occurrence of epidemic diseases in macroscopic level to the influences on organism cell properties in microscopic level. Although existing studies have debates over the biological effects of microwave, unanimous conclusions have been gradually made in thermal effects and non-thermal effects. Therefore, for promoting the deepening of microwave biological effects, it is urgent to constantly summarize present research progress. 


\section{References}

[1] Kaur G, Cameron-Smith D, Garg M, et al. Docosapentaenoic acid (22:5n-3): A review of its biological effects[J]. Progress in Lipid Research, 2011, 50(1):28-34.

[2] Scheuhammer A, Braune B, Chan H M, et al. Recent progress on our understanding of the biological effects of mercury in fish and wildlife in the Canadian Arctic[J]. Science of the Total Environment, 2015, 509-510:91-103.

[3] Zelník V, Lapuníková B, Kúdelová M. Marek' s disease: rapid progress in research with unclear biological implementations.[J]. Acta Virologica, 2013, 57(2):265-70.

[4] Gao L G, Luo F, Hui R T, et al. Recent molecular biological progress in Marfan syndrome and Marfan-associated disorders.[J]. Ageing Research Reviews, 2010, 9(3):363-368. 